\title{
Focus on Silodosin: Pros and Cons of Uroselectivity
}

This article was published in the following Dove Press journal:

Research and Reports in Urology

\author{
Gianluigi Califano' \\ Claudia Collà Ruvolo (D) \\ Massimiliano Creta $\mathbb{D}^{\prime}$ \\ Marco Capece (D) \\ Roberto La Rocca' \\ Giuseppe Celentano' \\ Luigi Napolitano $\mathbb{D}^{\prime}$ \\ Francesco Paolo Calace \\ Gianluca Spena (D) \\ Francesco Trama $\mathbb{D}^{\prime}$ \\ Felice Crocetto' \\ Francesco Mangiapia' \\ Nicola Longo' \\ Ferdinando Fusco ${ }^{2}{ }^{2}$ \\ 'Department of Neurosciences, \\ Reproductive Sciences and \\ Odontostomatology - Federico II \\ University of Naples, Naples, Italy; \\ ${ }^{2}$ Department of Urology, Luigi Vanvitelli \\ University of Naples, Naples, Italy
}

Correspondence: Massimiliano Creta

Department of Neurosciences,

Reproductive Sciences and

Odontostomatology, University of Naples

Federico II, via Sergio Pansini 5, Naples

$80|3|$, Italy

$\mathrm{Tel} / \mathrm{Fax}+3908 \mathrm{I} 74626 \mathrm{II}$

Email max.creta@gmail.com

\begin{abstract}
Medical management of lower urinary tract symptoms related to benign prostatic obstruction engages healthcare professionals worldwide. Currently, alpha-1 adrenergic antagonists are strongly recommended as first-line therapy for patients with moderate to severe symptoms because of their safety, efficacy and good tolerability. These agents are highly heterogeneous in terms of pharmacological selectivity for the adrenergic receptor subtypes with silodosin being the agent characterized by the highest $\alpha_{1 \mathrm{~A}} / \alpha_{1 \mathrm{~B}}$ affinity ratio. This property has been proposed to confer to silodosin advantages in terms of bladder outlet obstruction improvement and lower incidence of cardiovascular side effects at the cost of a higher incidence of ejaculatory dysfunction. These aspects should be carefully taken in consideration when personalizing medical therapy for lower urinary tract symptoms related to benign prostatic obstruction.
\end{abstract}

Keywords: alpha-1 adrenergic antagonists, benign prostate enlargement, lower urinary tract symptoms, bladder outlet obstruction

\section{Introduction}

Benign prostatic enlargement (BPE), is a common nonmalignant disorder affecting a growing number of men worldwide. ${ }^{1,2}$ The resulting benign prostatic obstruction (BPO), a condition defined on the basis of invasive urodynamic investigations as high-pressure/low-flow micturition pattern and diagnosed when the bladder outlet obstruction index (BOOI) is $>40$, has been traditionally considered the main pathophysiological link between BPE and lower urinary tract symptoms (LUTS). ${ }^{3-7}$ Moreover, pre-clinical and clinical evidences exist supporting the hypothesis that BPO can also lead to progressive morphological remodelling of the bladder and the upper urinary tract with subsequent potential serious functional impairments. ${ }^{3}$ Interestingly, increased intravesical pressure represents one of the most important stress factors stimulating molecular and cellular events involved in the pathogenesis of bladder wall remodelling following BPO. ${ }^{3}$ The management of patients with LUTS suggestive of BPO (LUTS/BPO) aims to ensure improvement of symptoms, quality of life (QoL) and, potentially, to prevent pathologic remodelling of the urinary tract. However, these patients are heterogeneous in terms of demographics, comorbidity profile, habits, needs, and expectations. Therefore, personalized treatments balancing desired effects against potential adverse events are strongly advocated.

\section{Alpha-I Adrenergic Antagonists (ABs) in the Treatment of LUTS/BPO Patients: The Concept of Uroselectivity}

Currently, $\mathrm{ABs}$ are strongly recommended as first-line therapy for patients with moderate to severe LUTS/BPO because of their safety, efficacy and good 
tolerability. ${ }^{5,8} \mathrm{ABs}$ represent a family of agents that bind to and inhibit type $1 \alpha$-adrenergic receptors (AR) thus counteracting the effects of endogenously released noradrenaline on smooth muscle cells in the lower urinary tract and improving the dynamic component of BPO. ${ }^{6}$ Nowadays, the following ABs are available in Europe: alfuzosin, doxazosin, silodosin, tamsulosin, and terazosin. ${ }^{5}$ These agents are heterogeneous in terms of "uroselectivity", a concept that may be discussed from different perspectives. ${ }^{4}$ Three $\alpha 1-A R$ subtypes $\left(\alpha_{1 \mathrm{~A}}\right.$, $\alpha_{1 \mathrm{~B}}$, and $\left.\alpha_{1 \mathrm{D}}\right)$ have been found in human tissues. The $\alpha_{1 \mathrm{~A}}$ subtype is the predominant subtype located in the human prostate, bladder base, bladder neck, prostatic capsule, and prostatic urethra, where it mediates the contraction of the smooth muscle cells. $\alpha_{1 \mathrm{D}}-\mathrm{ARs}$ are also expressed to a significant extent in the human prostate, and $\alpha_{1 \mathrm{~B}}$-ARs are mainly involved in the contraction of smooth muscle cells located in human arteries. Pharmacological uroselectivity, defines the selectivity toward $\alpha_{1 \mathrm{~A}}$ and $\alpha_{1 \mathrm{D}}$-AR versus $\alpha_{1 \mathrm{~B}^{-}}$ $\mathrm{AR} .{ }^{4}$ On the other hand, clinical uroselectivity can be defined as the balance between desired clinical effects on obstruction and symptoms vs unwanted side effects. ${ }^{4}$ The early ABs (alfuzosin, doxazosin, terazosin) are nonselective for AR subtype. Tamsulosin has relative selectivity for the $\alpha_{1 \mathrm{~A}}$ and $\alpha_{1 \mathrm{D}}$-subtypes. It is 15 - and 3-fold more selective for the $\alpha_{1 \mathrm{~A}^{-}}$ AR than for the $\alpha_{1 \mathrm{~B}}$ and $\alpha_{1 \mathrm{D}}$ subtypes, respectively. Silodosin is a new, highly selective $\mathrm{AB}$ characterized by a very strong affinity for the $\alpha_{1 \mathrm{~A}}-\mathrm{AR}$ (162 times higher than that for the $\alpha_{1 \mathrm{~B}}-\mathrm{AR}$, and 55 times higher than that for the $\left.\alpha_{1 \mathrm{D}}-\mathrm{AR}\right)$. From a clinical point of view, all ABs have been reported to provide statistically and clinically significant improvements in terms of both voiding and storage LUTS with respect to placebo, and to rise patients' QoL. Overall, there are only minimal differences in the efficacy of $\mathrm{ABs}$ in improving LUTS/BPO. Therefore, no specific recommendations are provided by officially recognized guidelines regarding the choice of which drug should be considered according to the patient's characteristics. ${ }^{6}$ However, evidences exist demonstrating clinical and urodynamic implications of pharmacological uroselectivity (Table 1).

\section{Pharmacological Uroselectivity Influences Urodynamic Efficacy of ABs}

The analysis of published evidence suggests the existence of a positive relationship between $\alpha_{1 \mathrm{~A}} / \alpha_{1 \mathrm{~B}}$ receptor affinity ratio and BOOI improvement. ${ }^{7}$ Indeed, when clustering $\mathrm{ABs}$ according to their receptor pharmacologic selectivity and
Table I Clinical and Urodynamic Implications of Pharmacological Uroselectivity

\begin{tabular}{|l|l|}
\hline Advantages & Disadvantages \\
\hline Higher mean BOOI improvement $^{7}$ & $\begin{array}{l}\text { Higher incidence of } \\
\text { EjDs }^{\prime \prime}\end{array}$ \\
\hline Higher mean Qmax improvement & \\
\hline Higher mean PdetQmax improvement & \\
\hline $\begin{array}{l}\text { Lower incidence of cardiovascular side } \\
\text { effects }\end{array}$ & \\
\hline
\end{tabular}

Abbreviations: $\mathrm{BOOI}$, bladder outlet obstruction index; EjDs, ejaculatory dysfunctions; PdetQmax, detrusor pressure at maximum urinary flow; Qmax, maximum urinary flow.

urodynamic efficacy, three subgroups can be identified with silodosin being the only member of a subgroup characterized by the highest levels of BOOI improvement and $\alpha_{1 \mathrm{~A}}-\mathrm{AR} / \alpha_{1 \mathrm{~B}}-$ AR affinity ratio. ${ }^{7}$ Accordingly, a systematic review and metaanalysis of studies evaluating the urodynamic outcomes of ABs in patients with LUTS/BPO showed a mean BOOI improvement of -14.88 for alfuzosin, -19.41 for doxazosin, 30.45 for silodosin, -14.27 for tamsulosin, and -6.69 for terazosin. ${ }^{6}$ Interestingly, BOOI reduction observed after therapy with $\mathrm{ABs}$ is due to a sustained decrease of mean detrusor pressure at maximum urinary flow (PdetQmax) and only to a marginal improvement of maximum urinary flow (Qmax). ${ }^{6}$ Magnitude of PdetQmax and Qmax improvement are higher after silodosin therapy with respect to other ABs. ${ }^{7}$ An explorative clinical study investigated the effects of silodosin $8 \mathrm{mg}$ once daily on obstruction urodynamic parameters and subjective symptoms in 30 Caucasian patients. ${ }^{9}$ All enrolled patients were obstructed (BOOI $>40$ ), reporting dissatisfaction with previous drug treatments and complained of severe symptoms for which they were awaiting surgery. The magnitude of BOOI improvement was both statistically and clinically significant, and $53.3 \%$ of patients improved their class according to BOOI (obstructed to unobstructed/obstructed to equivocal/equivocal to unobstructed). Also, total IPSS score, IPSS storage and voiding sub-scores as well as IPSS QoL sub-score improved in a statistically significant manner. Most interestingly, at the end of the treatment all patients declared that their condition improved enough to spare or delay surgery. ${ }^{9}$

\section{Pharmacological Uroselectivity Influences Adverse Events (AEs) Profile of ABs}

Differences in terms of prevalence of AEs have been reported among the different $\mathrm{ABs}$ and have been related to 
pharmacological uroselectivity. These effects commonly include dizziness, headache, postural hypotension, and ejaculatory dysfunctions (EjDs) (a broad spectrum of conditions ranging from absence of seminal emission, reduced ejaculate volume, and reduced ejaculation force). ${ }^{10-14}$ Non-selective drugs, such as doxazosin and terazosin are associated with higher incidence of cardiovascular AEs. ${ }^{15}$ A meta-analysis on the vascular-related safety of ABs showed that the odds of developing a cardiovascular-related $\mathrm{AE}$ were 3.71 for terazosin, 3.32 for doxazosin, 1.66 for alfuzosin, and 1.42 for tamsulosin as compared with placebo. ${ }^{16}$ The $\alpha_{1 \mathrm{~A}}$-AR selectivity profile of silodosin minimizes the propensity for blood pressure-related adverse effects caused by $\alpha_{1 \mathrm{~B}}$-AR blockade such as orthostatic hypotension, dizziness, and cardiovascular AEs. ${ }^{15}$ Novara et al analyzed the clustered datasets of the USA and European registrational trials to provide insights on the safety of silodosin. ${ }^{15}$ Dizziness and orthostatic hypotension had similar prevalence rates in the silodosin and placebo groups $\left(1.9 \%\right.$ vs $0.6 \%$ and $1.3 \%$ vs $1.1 \%$, respectively). ${ }^{15}$ Modification of systolic blood pressure, diastolic blood pressure, and heart rate, have also been reported to be similar between silodosin and placebo. ${ }^{11,15,16}$ Although $\alpha_{1 \mathrm{~A}}$ receptor selectivity is associated with less systemic undesirable effects, on the other side it results in a greater risk of disturbances in ejaculatory function. Indeed, the incidence of EjDs with ABs has been reported to increase with increasing $\mathrm{AB}$ specificity. In vitro studies on human vas deferens demonstrated that $\alpha \mathrm{AR}$ blockade eliminates electrically induced contractions, with the $\alpha_{1 \mathrm{~A}}$ subtype being the critical AR subtype involved in human vas deferens contractions. Therefore, a loss of seminal emission rather than retrograde ejaculation represents the main process behind the pathogenesis of EjDs. ${ }^{11,17}$ Based on evidence from clinical studies, the percentage of patients reporting EjDs was $0 \%, 0.3 \%$, and $3.6 \%$ after alfuzosin, terazosin, and doxazosin, respectively, with no statistically significant difference compared to placebo in blinded controlled trials involving doxazosin and terazosin. ${ }^{17}$ The incidence of EjDs with tamsulosin has been reported to range between $11 \%$ and $18 \% .{ }^{17}$ Of note, Novara et al found EjDs to be the most frequently reported adverse drug reaction in patients receiving silodosin $(22 \%$ patients in the silodosin group and $0.9 \%$ in the placebo group (OR 28.14; $\mathrm{P}<0.001)$ ). Interestingly, discontinuation rates due to adverse drug reactions in patients taking silodosin are low (4.3\%) and only $2 \%$ higher than with placebo. ${ }^{15}$ Moreover, evidence exists demonstrating that EjDs can even be considered predictors of therapeutic efficacy. ${ }^{10,12}$ Indeed, post hoc analyses of data from randomized controlled trials showed that patients experiencing EjD during treatment with silodosin were those with the highest improvements in terms of LUTS. Finally, the occurrence of EjDs seems to be related also with the progressive improvement of both urinary symptoms and flow rate over time. ${ }^{13}$

\section{Conclusions}

Silodosin is the member of the ABs family characterized by the highest $\alpha_{1 \mathrm{~A}}-\mathrm{AR} / \alpha_{1 \mathrm{~B}}-\mathrm{AR}$ affinity ratio. This unique pharmacological profile has relevant urodynamic and clinical implications. Pros of pharmacological uroselectivity profile that characterize silodosin among other $\mathrm{ABs}$ include highest level of BOOI improvement and placebolike cardiovascular AE. These advantages are achieved at the cost of highest rate of EjDs. The safety profile of silodosin makes it a useful agent for the management of patients suffering from low blood pressure and for those receiving therapy with hypotensive agents. Finally, remembering its ability to delay the need for surgery, is to be highlighted the impact it could have on the reduction of surgical waiting lists. In young and sexually active men, the risk of EjDs deserves careful discussion.

\section{Abbreviations}

Abs, alpha-1 adrenergic antagonists; AE, adverse events; $\mathrm{AR}$, adrenergic receptor; BPE, benign prostatic enlargement; BPO, benign prostatic obstruction; EjDs, ejaculatory Dysfunctions; LUTS, lower urinary tract symptoms; BOOI, bladder outlet obstruction index; PVR, postvoid residual urine volume; QoL, quality of life.

\section{Disclosure}

Ferdinando Fusco is speaker for Recordati and reports grants, personal fees from Recordati. Massimiliano Creta is a speaker and medical writer for Recordati and reports grants, personal fees from Recordati SPA, personal fees, non-financial support from IBSA, during the conduct of the study. The authors report no other conflicts of interest in this work.

\section{References}

1. Creta M, Bottone F, Sannino S, et al. Effects of alpha1-blockers on urodynamic parameters of bladder outlet obstruction in patients with lower urinary tract symptoms suggestive of benign prostatic enlargement: a review. Minerva Urol Nefrol. 2015.

2. Osman NI, Chapple CR, Cruz F, Desgrandchamps F, Llorente C, Montorsi F. Silodosin: a new subtype selective alpha-1 antagonist for the treatment of lower urinary tract symptoms in patients with benign prostatic hyperplasia. Expert Opin Pharmacother. 2012;13 (14):2085-2096. doi:10.1517/14656566.2012.714368 
3. Fusco F, Creta M, De Nunzio C, et al. Progressive bladder remodeling due to bladder outlet obstruction: a systematic review of morphological and molecular evidences in humans. BMC Urol. 2018;18 (1):15. doi:10.1186/s12894-018-0329-4

4. Korstanje C, Krauwinkel W, van Doesum-wolters FL. Tamsulosin shows a higher unbound drug fraction in human prostate than in plasma: a basis for uroselectivity? Br J Clin Pharmacol. 2011;72 (2):218-225. doi:10.1111/j.1365-2125.2010.03870.x

5. Gratzke C, Bachmann A, Descazeaud A, et al. EAU guidelines on the assessment of non-neurogenic male lower urinary tract symptoms including benign prostatic obstruction. Eur Urol. 2015;67 (6):1099-1109. doi:10.1016/j.eururo.2014.12.038

6. Fusco F, Palmieri A, Ficarra V, et al. $\alpha 1$-blockers improve benign prostatic obstruction in men with lower urinary tract symptoms: a systematic review and meta-analysis of urodynamic studies. Eur Urol. 2016;69(6):1091-1101. doi:10.1016/j.eururo.2015.12.034

7. Fusco F, Creta M, Imperatore V, et al. Benign prostatic obstruction relief in patients with lower urinary tract symptoms suggestive of benign prostatic enlargement undergoing endoscopic surgical procedures or therapy with alpha-blockers: a review of urodynamic studies. Adv Ther. 2017;34(4):773-783. doi:10.1007/s12325-017-0504-0

8. Oelke M, Bachmann A, Descazeaud A, et al. EAU guidelines on the treatment and follow-up of non-neurogenic male lower urinary tract symptoms including benign prostatic obstruction. Eur Urol. 2013;64 (1):118-140. doi:10.1016/j.eururo.2013.03.004

9. Fusco F, Creta M, Longo N, Persico F, Franco M, Mirone V. Silodosin $8 \mathrm{mg}$ improves benign prostatic obstruction in Caucasian patients with lower urinary tract symptoms suggestive of benign prostatic enlargement: results from an explorative clinical study. BMC Urol. 2018;18(1):12. doi:10.1186/s12894-018-0326-7

10. Roehrborn CG, Kaplan SA, Lepor H, Volinn W. Symptomatic and urodynamic responses in patients with reduced or no seminal emission during silodosin treatment for LUTS and BPH. Prostate Cancer Prostatic Dis. 2011;14(2):143-148. doi:10.1038/pcan.2010.46
11. Chapple CR, Montorsi F, Tammela TL, Wirth M, Koldewijn E, Fernández Fernández E. Silodosin therapy for lower urinary tract symptoms in men with suspected benign prostatic hyperplasia: results of an international, randomized, double-blind, placebo- and active-controlled clinical trial performed in Europe. Eur Urol. 2011;59(3):342-352. doi:10.1016/j.eururo.2010.10.046

12. Homma Y, Kawabe K, Takeda M, Yoshida M. Ejaculation disorder is associated with increased efficacy of silodosin for benign prostatic hyperplasia. Urology. 2010;76(6):1446-1450. doi:10.1016/j. urology.2010.03.015

13. Gacci M, Ficarra V, Sebastianelli A, et al. Impact of medical treatments for male lower urinary tract symptoms due to benign prostatic hyperplasia on ejaculatory function: a systematic review and meta-analysis. J Sex Med. 2014;11(6):1554-1566. doi:10.1111/ jsm. 12525

14. Capitanio U, Salonia A, Briganti A, Montorsi F. Silodosin in the management of lower urinary tract symptoms as a result of benign prostatic hyperplasia: who are the best candidates. Int J Clin Pract. 2013;67(6):544-551. doi:10.1111/ijcp.12135

15. Novara G, Chapple CR, Montorsi F. A pooled analysis of individual patient data from registrational trials of silodosin in the treatment of non-neurogenic male lower urinary tract symptoms (LUTS) suggestive of benign prostatic hyperplasia (BPH). BJU Int. 2014;114 (3):427-433. doi:10.1111/bju. 12712

16. Nickel JC, Sander S, Moon TD. A meta-analysis of the vascular-related safety profile and efficacy of alpha-adrenergic blockers for symptoms related to benign prostatic hyperplasia. Int J Clin Pract. 2008;62 (10):1547-1559. doi:10.1111/j.1742-1241.2008.01880.x

17. Welliver C, Butcher M, Potini Y, McVary KT. Impact of alpha blockers, 5-alpha reductase inhibitors and combination therapy on sexual function. Curr Urol Rep. 2014;15(10):441. doi:10.1007/ s11934-014-0441-2

\section{Publish your work in this journal}

Research and Reports in Urology is an international, peer-reviewed, open access journal publishing original research, reports, editorials, reviews and commentaries on all aspects of adult and pediatric urology in the clinic and laboratory including the following topics: Pathology, pathophysiology of urological disease; Investigation and treatment of urological disease; Pharmacology of drugs used for the treatment of urological disease. The manuscript management system is completely online and includes a very quick and fair peer-review system, which is all easy to use. Visit http://www.dovepress.com/ testimonials.php to read real quotes from published authors 